\title{
Straight-to-Curvilinear Motion Transition of a Swimming Droplet Caused by the Susceptibility to Fluctuations
}

\section{$\operatorname{AUTHOR}(\mathrm{S}):$}

Suda, Saori; Suda, Tomoharu; Ohmura, Takuya; Ichikawa, Masatoshi

\section{CITATION:}

Suda, Saori ... [et al]. Straight-to-Curvilinear Motion Transition of a Swimming Droplet Caused by the Susceptibility to Fluctuations. Physical Review Letters 2021, 127(8): 088005.

\section{ISSUE DATE:}

2021-08

URL:

http://hdl.handle.net/2433/265074

\section{RIGHT:}

(c) 2021 American Physical Society; 許諾条件に基づいて掲載しています $\circ$ 


\title{
Straight-to-Curvilinear Motion Transition of a Swimming Droplet Caused by the Susceptibility to Fluctuations
}

\author{
Saori Suda, ${ }^{1}$ Tomoharu Suda $\odot,{ }^{2}$ Takuya Ohmura $\odot,{ }^{3, *}$ and Masatoshi Ichikawa ${ }^{1}$ \\ ${ }^{1}$ Department of Physics, Kyoto University, Kyoto 606-8502, Japan \\ ${ }^{2}$ Department of Mathematics, Keio University, Yokohama 223-8522, Japan \\ ${ }^{3}$ Max Planck Institute for Terrestrial Microbiology, 35043 Marburg, Germany
}

(Received 14 October 2020; revised 11 June 2021; accepted 24 June 2021; published 19 August 2021)

In this Letter, a water-in-oil swimming droplet's transition from straight to curvilinear motion is investigated experimentally and theoretically. An analysis of the experimental results and the model reveal that the motion transition depends on the susceptibility of the droplet's direction of movement to external stimuli as a function of environmental parameters such as droplet size. The simplicity of the present experimental system and the model suggests implications for a general class of transitions in self-propelled swimmers.

DOI: 10.1103/PhysRevLett.127.088005

At the end of the 19th century, G. Quincke presented self-propelled droplets as a model system for a migrating cell in an aqueous environment $[1,2]$. He stated that the fluid convection inside and outside of the swimming droplet is similar to that observed in the protoplasmic streaming of the amoeba cell. Today, scientists understand that the droplet systems are not living cells themselves because their chemical systems are significantly different. However, physical and fluid-dynamical essentialities show common characteristics with both systems, e.g., roll convection, and their behaviors should be explored further with the fluid dynamics of a self-propelled object [3-8], the physics of collective motions $[9,10]$, and the force balance of adhesion and surface tension under nonequilibrium conditions [11].

In recent decades, there has been an increased interest in the spatiotemporal organization of self-propelled elements called active matters, e.g., swarms of fish, birds, animals, and microorganisms. It is expected that the recognition of the physical phenomena that are unique to spontaneous and collective motions can be applied to various fields involving statistical physics, chemical technology, and life sciences $[9,12,13]$. Swimming droplets have been reexamined as an artificial active matter system with the special property of their motility being controllable using chemical means [10]. The motion of droplets induced by the Marangoni effect and wetting phenomena has been studied from the viewpoint of fundamental sciences $[14,15]$ and reaction control engineering [16-18]. While the driving forces in these systems have been actively studied, understanding motion controls such as chemotactic motion [19] and spontaneous changes in motility remains a challenging task. Additionally, swimming droplets are still significant as models of the single and collective motion of living organisms. For example, various "living" motions are observed even in individual droplets [10,20-24]. However, the emergence of motion diversity is not clearly understood.

Several examples of the motilities of swimming droplets have been reported. Liquid crystals enable us to extract a specific mode of motion by controlling the symmetry inside the droplets [25-28], where straight, curvilinear, and spiral motions, and their transitions, have been demonstrated. As a simpler case, spontaneous symmetry breaking of the motion of a swimming droplet in a two-dimensional (2D) system was investigated experimentally [29,30] and theoretically [31], which identified the straight-to-curvilinear motion transition. Recent experimental studies reported that an increase of the external surfactant concentration or the viscosity of the swimming medium causes the curvilinear motion of a swimming spherical droplet [22,23]. In addition, the previous studies showed the existence of a relation between the emergence of the complex motion and the onset of higher hydrodynamic modes [23,32]. However, the exact connection is not yet explicitly confirmed.

In our study, we measured the motion and internal flow of a spherical swimming water microdroplet and quantitatively identified a straight-to-curvilinear motion transition. To reveal the relationship between the curvilinear motion and the higher hydrodynamic modes, we developed a 3D axis-asymmetric (torque-free) model consisting of the advection-diffusion equation, with influx and outflux of surfactants, coupled with the Stokes equation and investigated it analytically.

Experiments were conducted following the previous study [21]. The individual droplets continued an active swimming motion for more than 30 minutes. Water is solubilized by reverse micelles in the oil phase [Fig. 1(a)]. The droplets are driven by the Marangoni effect, induced by the inhomogeneity of the surface tension or surface coverage of the surfactants, which correlates to the 

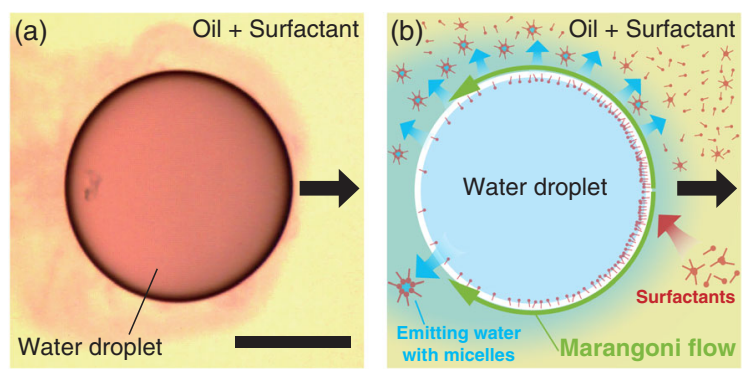

FIG. 1. (a) Solubilization of water around a swimming droplet. The water is dyed using Acid Red 52 (Tokyo Chemical Industry Co., Ltd.). The red color gradient around the droplet shows that the stained water inside the droplet was being emitted. The black arrow represents the direction of the droplet's movement. The scale bar represents $200 \mu \mathrm{m}$. (b) Schematic illustration of the swimming mechanism of a droplet. The droplet is driven by the Marangoni flow (green arrows) generated by the solubilization of water (blue arrows).

solubilization rate and the concentration of the swollen reverse micelles nearby $[21,33]$. A schematic illustration of the swimming mechanism is shown in Fig. 1(b). See A in the Supplemental Material [34], which includes Ref. [35], for details of the experimental setup. In Fig. 2(a), typical trajectories of the centroids of the droplets are represented for three different radii scales. Figure 2(b) displays the trajectories of the centroids of the droplets for $30 \lesssim R \lesssim 400 \mu \mathrm{m}$.

The droplets tend to take more curved trajectories as their radii increase. To confirm this observation quantitatively, we calculated the angular autocorrelation function $\langle C(t, \tau)\rangle_{t}$ of the direction of movement of the droplets
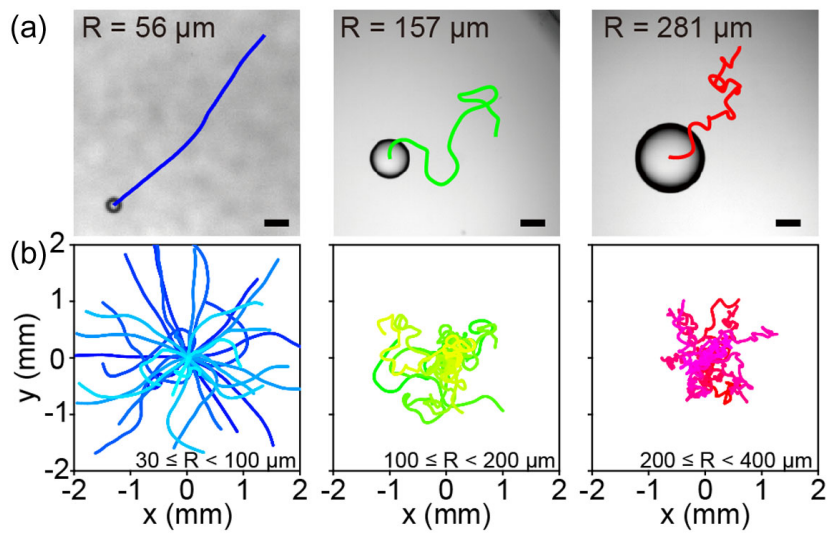

FIG. 2. Trajectories of the centroids of the swimming droplets over 60 seconds (see videos 1-3 in the Supplemental Material). (a) The typical trajectories of the droplet centroids. The radii of the water droplets are $56 \mu \mathrm{m}$ (left), $157 \mu \mathrm{m}$ (middle), and $281 \mu \mathrm{m}$ (right). The scale bars represent $200 \mu \mathrm{m}$. (b) Trajectories of the centroids of the droplets for three categories of droplet radii. The starting points are set to the origin. The radii categories are $30 \leq$ $R<100 \mu \mathrm{m}$ (left), $100 \leq R<200 \mu \mathrm{m}$ (middle), and $200 \leq R<$ $400 \mu \mathrm{m}$ (right). Color variation depicts different droplets. with velocity $\mathbf{v}(t)$, which is given as a function of lag time $\tau:\langle C(t, \tau)\rangle_{t}=\left\langle[\mathbf{v}(t+\tau) \cdot \mathbf{v}(t)] /|\mathbf{v}(t+\tau)||\mathbf{v}(t)|>_{t}\right.$, where \langle\rangle$_{t}$ represents the time average, and a three-point moving average was applied to the velocity [Fig. 3(a)]. The fastest relaxation time attained $\sim 1.4 \mathrm{~s}$ [Fig. 3(b)], and thus $\tau_{\min }=1.4 \mathrm{~s}$ was a suitable lag time to identify the degree of curved motion as $\arccos C\left(t, \tau_{\min }\right)$. Qualitatively similar behavior has been observed in other experimental systems $[22,23]$. The oscillatory motion of the large droplets is possibly related to the chaotic motion shown in the previous study [32]. To quantify the persistence of the straight motion, we introduced the decay time of $\langle C(t, \tau)\rangle_{t}$ by fitting it with the angular autocorrelation function of the Brownian harmonic oscillator $\langle C(t, \tau)\rangle_{t}=e^{-a_{1} \tau+a_{2}} \cos a_{3} \tau$ [36]. The decay time $1 / a_{1}$ was plotted as a function of the droplet radius, which indicated that the decay times tend to change at approximately $R \approx 100 \mu \mathrm{m}$ [Fig. 3(b)]. The average speed increased with the radius and saturated at $R \approx 100 \mu$ m [Fig. 3(c)].

The flow inside the droplets in the equatorial plane was measured via particle imaging velocimetry (PIV). Besides dipolar flow, quadrupolar flow was observed for some droplets with $R \gtrsim 200 \mu \mathrm{m}$ when they turned (shown in B of the Supplemental Material). Furthermore, the PIV experiment evaluated contributions of the flow modes [Fig. 4(b)] and especially the difference angle between the axes of dipolar and quadrupolar flow fields, which corresponds to $\Psi / 2$ as we see later in this analysis. The systematic correlation between the difference angle $\Psi$ and the turning angle of the movement of the droplet [Fig. 4(a)] suggests that the angle plays an essential role in the transition of the motion.

To validate this hypothesis and explain the mechanism of the transition, we developed a 3D axis-asymmetric theoretical model, based on a theoretical study of a swimming droplet driven by the Marangoni flow in a 2D system [31].
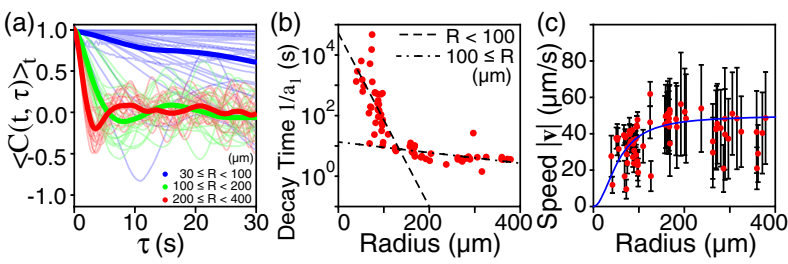

FIG. 3. Analysis of the experimental results. (a) Angular autocorrelation function $\langle C(t, \tau)\rangle_{t}$ of the direction of movement of the droplets. The thin lines correspond to the individual droplets, and the thick lines show the ensemble averages of the droplets in the respective size categories. (b) Droplet radius dependence of the decay time $1 / a_{1}$. Each black dashed line represents an exponential fitting line for the data points with $R<100 \mu \mathrm{m}$ and $\geq 100 \mu \mathrm{m}$. (c) Droplet radius dependence of the time-averaged speed of the droplets (red dots). Error bars represent the standard deviations of the speed. The blue line is a fitting curve using Eq. (26) in $\mathrm{H}$ of the Supplemental Material with $R<250 \mu \mathrm{m}$. 

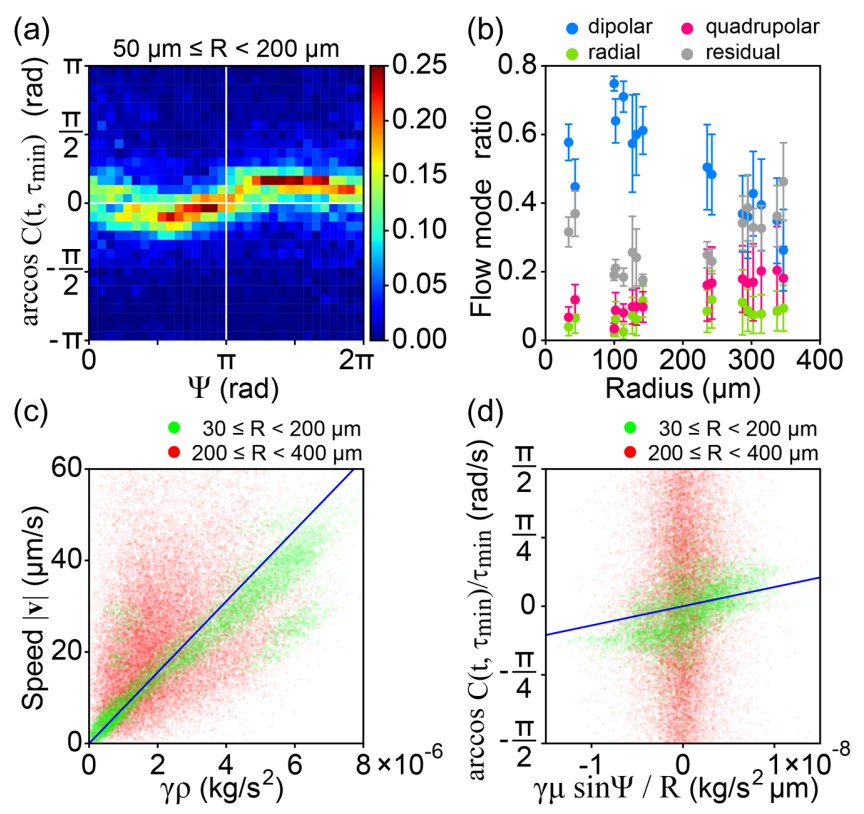

FIG. 4. Results of PIV experiments. The flow modes were extracted from PIV data of the internal flow and were calculated into the concentration mode of the chemical. (a) 2D density map between the difference angle $\Psi=\lambda-2 \nu$ and the degree of curved motion as $\arccos C\left(t, \tau_{\min }\right)$. The color indicates the density. (b) The average ratio of flow modes observed in the PIV experiments, i.e., $(l, m)=(1, \pm 1)$ : dipolar, $(2,0)$ : radial, $(2, \pm 2)$ : quadrupolar, and the residual flow fields, given by the norm of each vector field, and the droplet radius. Error bars represent the standard deviations of the ratios. (c) Scatter diagram between the speed of the droplet $|\mathbf{v}|$ and model suggested parameter $\gamma \rho$ estimated from the PIV results. The green dots represent $30<R<200 \mu \mathrm{m}$ and red dots represent $200 \leq R<$ $400 \mu \mathrm{m}$. The model denotes the blue line as $|\mathbf{v}|=7.77 \times$ $10^{6} \times \gamma \rho \mu \mathrm{m} \mathrm{s}^{-1}$. (d) Scatter diagram between angular velocity $\arccos C\left(t, \tau_{\min }\right) / \tau_{\min }$ and $\gamma \mu \sin \Psi / R$. The green and red dots represent the same range as those of (c). The blue line is predicted by the present model without free fitting parameters as $d \nu / d t=2.20 \times 10^{7} \times(\gamma \mu \sin \Psi / R) \mathrm{rad} \mathrm{s}^{-1}$.

The velocity of the droplets was assumed to lie in the horizontal plane in agreement with the experimental observations. In constructing the model, we considered the flow on the surface of the droplets instead of the internal flow, which is determined by the surfactant concentration on the droplet surface [6].

The surfactant concentration $c(\theta, \phi)$ on the droplet surface, parametrized by the polar angle $\theta$ and azimuthal angle $\phi$, is assumed to obey

$\frac{\partial c}{\partial t}+\mathbf{u} \cdot \nabla c=\frac{D}{R^{2}} \Delta_{\text {sphere }} c-\alpha c+\alpha \beta \delta\left(\theta-\frac{\pi}{2}, \phi-\arg \frac{\mathbf{v}}{|\mathbf{v}|}\right)$,

where $\alpha$ and $\beta$ are the characteristic duration of stay on the surface and the relative rate of the supply of the surfactant, $\mathbf{u}$ is the flow field on the droplet surface, $D$ is the diffusion coefficient of the surfactant on the surface, and

$$
\Delta_{\text {sphere }}=\frac{1}{\sin \theta} \frac{\partial}{\partial \theta}\left(\sin \theta \frac{\partial}{\partial \theta}\right)+\frac{1}{\sin ^{2} \theta} \frac{\partial^{2}}{\partial \phi^{2}}
$$

is the Laplacian operator on the unit sphere. A detailed discussion of the current model setup is provided in $\mathrm{C}$ of the Supplemental Material, which includes Refs. [37-39].

We expand the surfactant concentration $c$ and the flow field $\mathbf{u}$ in terms of spherical harmonics $Y_{l}^{m}$ using the solution of the Stokes equation in terms of the surface tension distribution (given in D of the Supplemental Material) [6]. The terms with lower-order spherical harmonics are retained so that the flow field inside the droplet is symmetrical in the horizontal plane, as the droplet was not moving significantly in the vertical direction and our focus here is the angle between the axes of dipolar and quadrupolar flows. Thus, we expand $c$ as where $c_{1}^{-1}=$ $-\left(c_{1}^{1}\right)^{*}$ and $c_{2}^{-2}=-\left(c_{2}^{2}\right)^{*}$ are complex numbers, and $c_{2}^{0}$ is a real number. On the equatorial plane, the term with $(l, m)=(1, \pm 1)$ yields a dipolar flow, $(2,0)$ a radial flow, and $(2, \pm 2)$ a quadrupolar flow (streamlines are shown in $\mathrm{E}$ of the Supplemental Material). We set the retained terms as $c_{1}^{1}=\rho e^{i \nu}, c_{2}^{0}=Z$, and $c_{2}^{2}=\mu e^{i \lambda}$, where $\rho>0$ and $\mu>0$. The variables $\rho$ and $\pi-\nu$ correspond to the strength and the direction of the "dipolar flow," respectively. Further, $\rho$ and $\nu$ determine the velocity of the droplet [6] as follows:

$$
\mathbf{v}=\frac{1}{3} \sqrt{\frac{6}{\pi}} \frac{\gamma \rho}{2 \eta+3 \hat{\eta}}\left(\begin{array}{c}
-\cos \nu \\
\sin \nu \\
0
\end{array}\right),
$$

where $\eta$ is the oil viscosity, $\hat{\eta}$ is the water viscosity, and $\gamma$ is a factor of proportionality between surface tension and concentration. Similarly, $\mu$ and $\pi-\lambda / 2$ correspond to the strength and the direction of the "quadrupolar flow."

From Eq. (1) and the solution of the Stokes equations, we obtain a system of ordinary differential equations governing the motion of the droplet (the derivation is described in F of the Supplemental Material). Nondimensionalization yields a system of equations:

$$
\begin{aligned}
\frac{d \bar{\rho}}{d \bar{t}}= & -\frac{3}{10 \bar{R}} \sqrt{\frac{5}{\pi}}(1+\chi)[-\sqrt{6} \bar{\rho} \bar{\mu} \cos (\lambda-2 \nu)+\bar{\rho} \bar{Z}] \\
& -\left(1+\frac{2 L^{2}}{\bar{R}^{2}}\right) \bar{\rho}+\frac{1}{4} \sqrt{\frac{6}{\pi}}, \\
\frac{d \nu}{d \bar{t}}= & \frac{3}{10 \bar{R}} \sqrt{\frac{30}{\pi}}(1+\chi) \bar{\mu} \sin (\lambda-2 \nu), \\
\frac{d \bar{Z}}{d \bar{t}}= & \frac{1}{35 \bar{R}} \sqrt{\frac{5}{\pi}}\left(7 \bar{\rho}^{2}+15 \chi \bar{Z}^{2}-30 \chi \bar{\mu}^{2}\right)-\left(1+\frac{6 L^{2}}{\bar{R}^{2}}\right) \bar{Z} \\
& -\frac{1}{4} \sqrt{\frac{5}{\pi}},
\end{aligned}
$$




$$
\begin{aligned}
\frac{d \bar{\mu}}{d \bar{t}}= & \frac{1}{40} \sqrt{\frac{30}{\pi}}\left(-\frac{4 \bar{\rho}^{2}}{\bar{R}}+5\right) \cos (\lambda-2 \nu)-\frac{6}{7 \bar{R}} \sqrt{\frac{5}{\pi}} \chi \bar{Z} \bar{\mu} \\
& -\left(1+\frac{6 L^{2}}{\bar{R}^{2}}\right) \bar{\mu} \\
\frac{d \lambda}{d \bar{t}}= & -\frac{1}{40} \sqrt{\frac{30}{\pi}}\left(-\frac{4 \bar{\rho}^{2}}{\bar{R}}+5\right) \frac{\sin (\lambda-2 \nu)}{\bar{\mu}}
\end{aligned}
$$

where the overlined symbols denote nondimensionalized quantities. The parameter $L=l_{d} / l_{a}$ is determined by constants $l_{d}=\sqrt{D / \alpha}$ and $l_{a}=\gamma \beta / \alpha(2 \eta+3 \hat{\eta})$, corresponding to the characteristic lengths of diffusion and advection, respectively. The parameter $\chi=(2 \eta+3 \hat{\eta}) /$ $(5 \eta+5 \hat{\eta})$ is calculated as 0.41 using the values of the viscosity of squalene $28.33 \times 10^{-3} \mathrm{~kg} /(\mathrm{m} \cdot \mathrm{s})$ [40] and water $0.89 \times 10^{-3} \mathrm{~kg} /(\mathrm{m} \cdot \mathrm{s})\left(0.1 \mathrm{MPa}, 25^{\circ} \mathrm{C}\right)$.

To quantitatively verify the consistency between the model and the experimental results, we estimated the surfactant concentration in the surface $c_{l}^{m}$ from the observed flow field by fitting the PIV results of the internal flow with the solution of the Stokes equation in terms of $\rho, \nu, Z, \mu$, and $\lambda$. Details of the fitting method are in $\mathrm{G}$ of the Supplemental Material. We reconstructed the vector fields using the variables $c_{1}^{1}=\rho e^{i \nu}, c_{2}^{0}=Z$, and $c_{2}^{2}=\mu e^{i \lambda}$, and the residual flow field was defined by the difference between the reconstruction and the observation. The relative strength of each term was estimated from the norm of each vector field [Fig. 4(b)]. The residual was large when $R \lesssim 50 \mu \mathrm{m}$, possibly caused by the insufficient resolution of the image. When $R \gtrsim 50 \mu \mathrm{m}$, as the droplet radius increased, the quadrupolar terms $(2,0)$ and $(2, \pm 2)$ became dominant, while the dipolar term $(1, \pm 1)$ diminished. The residual also increased due to higher-order terms.

Speeds of the droplets in the experiments are compared with ones predicted by Eq. (2) using values of $\gamma \rho$ estimated from the PIV results [Fig. 4(c)]. The speed was observed to be proportional to $\gamma \rho$ for $R<200 \mu \mathrm{m}$ (green dots), distributed along the blue line representing the model with the same viscosity values as above.

Angular velocities are also estimated from the dimensional version of Eq. (3b). We obtain a relation

$$
\frac{d \nu}{d t}=\frac{3}{50} \sqrt{\frac{30}{\pi}} \frac{7 \eta+8 \hat{\eta}}{(\eta+\hat{\eta})(2 \eta+3 \hat{\eta})} \frac{\gamma \mu \sin \Psi}{R},
$$

where $\Psi=\lambda-2 \nu$. Figure 4(d) displays a scatter plot between the angular velocity of the motion direction vector of the droplet in adopting $\arccos C\left(t, \tau_{\min }\right) / \tau_{\min }$ and $\gamma \mu \sin \Psi / R$ evaluated from the PIV experiments. The distribution normalized by $\gamma \mu \sin \Psi / R$ for $R<200 \mu \mathrm{m}$ (green dots) suggests a master curve, and that is represented by the above equation without free fitting parameters (blue line), i.e., the model is consistent with the PIV result.

To investigate the possible scenarios of the motion transition, we conducted a linear stability analysis for (a-1)
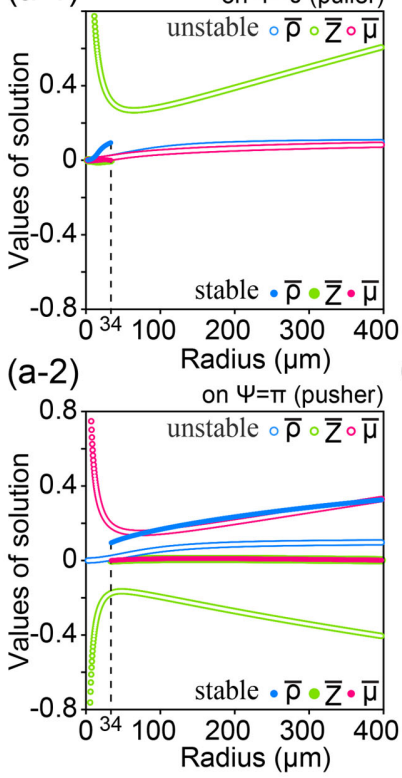

(b)

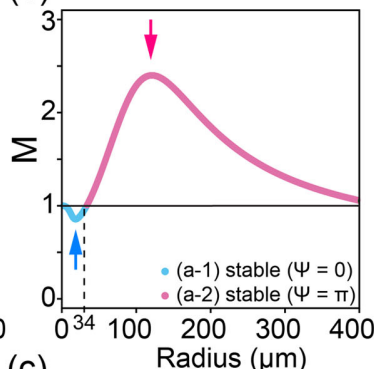

(c)

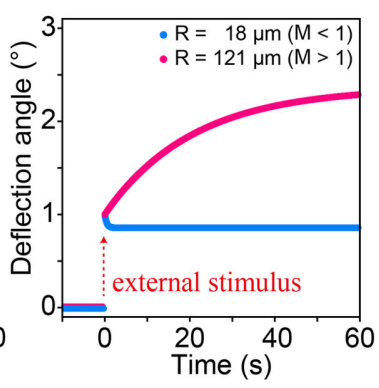

(d-1) $M<1$
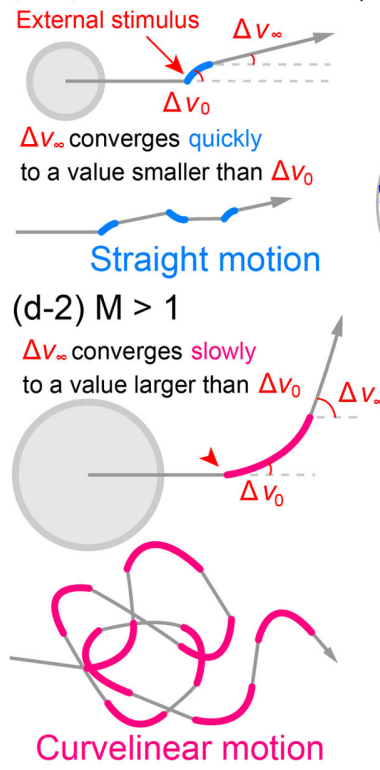

(e)

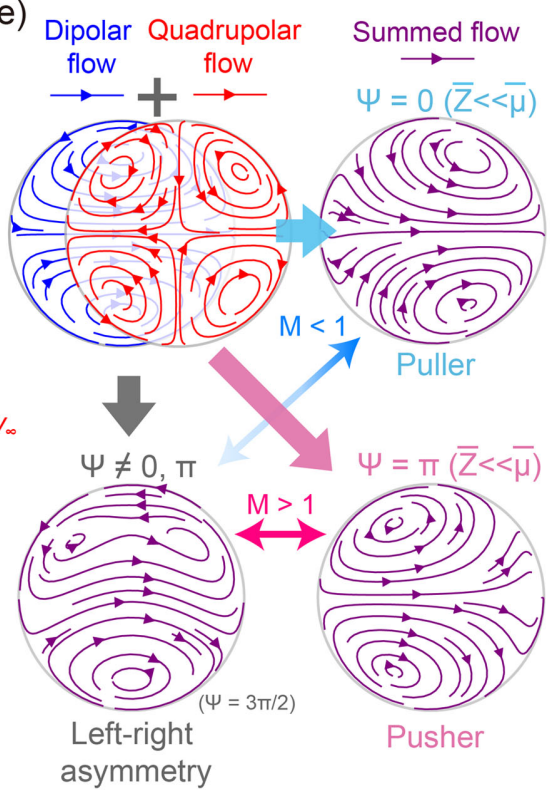

FIG. 5. Analysis of solutions of straight motion for the diffusion coefficient $D=10^{-10} \mathrm{~m}^{2} / \mathrm{s}$. (a) Values and stability of solutions of $\Psi=0$ : puller (a-1) and $\Psi=\pi$ : pusher (a-2). (b) Multiplier coefficient $M$ of motion direction against stimulus versus droplet radius $R$. (c) Time development of the direction of movement of the droplet from the initial direction when the external stimulus $1^{\circ}$ is applied to the direction of movement. For the stable solution of straight motion at radius $R=18 \mu \mathrm{m}$ (blue) and $R=121 \mu \mathrm{m}$ (magenta). (d) Schematic illustrations of motions of droplets under stimuli in real space with $M<1$ (d-1) and $>1$ (d-2). (e) Schematic diagram of the internal flow modes of the droplet. 
Eq. (3). Solutions of stable straight motion should satisfy $d \nu / d \bar{t}=0$, i.e., $\sin \Psi=0$. This is further classified into two cases $\Psi=0$ and $\Psi=\pi$. Using the effective squirmer parameter

$$
\frac{3 \sqrt{3}}{20 \bar{\rho}}(\sqrt{6} \bar{\mu} \cos \Psi-\bar{Z}),
$$

we see that $\Psi=0$ and $\Psi=\pi$ correspond to puller and pusher squirmer types, respectively, if $\bar{Z}$ is small [6]. Examples of flow fields are shown in $I$ of the Supplemental Material. For $\Psi=0$ and $\Psi=\pi$, we numerically calculated $\bar{\rho}, \bar{Z}, \bar{\mu}$ of Eq. (3) and the eigenvalues of the coefficient matrices of the linearized systems. A result consistent with the experiment is obtained using the value $D=10^{-10} \mathrm{~m}^{2} / \mathrm{s}$. There are stable solutions with $\Psi=0$ for $R \lesssim 34 \mu \mathrm{m}$ [Fig. 5(a-1)] and with $\Psi=\pi$ for $R \gtrsim 34 \mu \mathrm{m}$ [Fig. 5(a-2)]. Although both are stable, the growth of the perturbation is different. The rate of increase of the deviation from the solution is defined by $\Delta \nu_{\infty}=M \Delta \nu_{0}$ with a stimulus $\Delta \nu_{0}$ and the following response $\Delta \nu_{\infty}$. The coefficient $M$ is analytically calculated as $M=$ $A_{2} /\left(A_{2}-2 A_{1}\right)$ where $d \nu / d \bar{t}=A_{1} \sin \Psi$ and $d \lambda / d \bar{t}=$ $A_{2} \sin \Psi$ are from Eqs. (3b) and (3e) (see $\mathrm{K}$ of the Supplemental Material). Figure 5(b) shows the coefficient $M$ at a stable solution of straight motion as a function of the droplet radius. If $M<1$, an effect of an external stimulus $\Delta \nu_{0}$ is rapidly damped and a straight motion restarts. If $M>1$, the effect leaves an imprint on the direction of movement for a longer time, and it gradually turns toward $\sim M \Delta \nu_{0}$. Actually, a numerical simulation of Eq. (3) confirms the significantly longer relaxation time in the case of $M>1$ than $M<1$, and total deflection angles in droplet movements are magnified by $\sim M$ [Fig. 5(c)]. Time developments of the respective motions under stimuli will differ phenomenologically [Fig. 5(d)].

This responsiveness transition from $M<1$ to $M>1$ occurs around the radius $R \approx 34 \mu \mathrm{m}$ as a result of the change of swimming mode between puller and pusher for the stable straight solution [Fig. 5(a)]. The size of the droplet is one of essential parameters to elevate the strengths of quadrupolar flow and concentration; therefore the responsiveness changes due to the droplet size variation. Since deviation of $\Psi$ from the solutions 0 and $\pi$ corresponds to left-right asymmetrical flow [Fig. 5(e)], effective periods exhibiting the asymmetrical flow accompanied by turning motion are enlarged by the property $M>1$ [Fig. 5(d)]. Apart from external stimuli such as thermal fluctuation, an internal perturbation is possibly induced by the self-generated chemical ununiformity [23]. From the above discussion, we conclude that the observed motion transition is precisely the transition of the angular response to perturbations.

There are two fundamental transitions observed in selfpropelled objects: stationary to moving and straight to curvilinear [22,23,29-31,33]. In particular, the latter transition is essential to the motion diversity. We experimentally and theoretically revealed that even an isotropic swimming droplet, which has spherical symmetry, exhibits a straight-to-curvilinear motion transition. Considering the simplicity of the experimental system and the present model, it is probable that similar orbital instability in straight swimming movement exists in a phase separation [15] and in a living cell such as an amoeboid swimmer [13,41-43]. Further study in this line would be of interest, as it may reveal a mechanism behind the rich variety of living and active motions in nature.

We would like to express our gratitude to Professor Olivier Dauchot, Dr. Charlotte de Blois, Professor Hiroyuki Kitahata, and Professor Yutaka Sumino for their useful critique of this research. This study was supported by Grant-in-Aid for JSPS Fellows (20J15804) and KAKENHI No. $21 \mathrm{~K} 03855$.

"Present address: Biozentrum, University of Basel, 4056 Basel, Switzerland.

[1] G. Quincke, Ann. Phys. (Berlin) 271, 580 (1888).

[2] B. Otto, Untersuchungen über mikroskopische Schäume und das Protoplasma; Versuche und Beobachtungen zur Lösung der Frage nach den physikalischen Bedingungen der Lebenserscheinungen. (Wilhelm Engelmann, Leipzig, 1892), p. 262.

[3] Y. Nishigami, M. Ichikawa, T. Kazama, R. Kobayashi, T. Shimmen, K. Yoshikawa, and S. Sonobe, PLoS One 8, e70317 (2013).

[4] M. J. Lighthill, Commun. Pure Appl. Math. 5, 109 (1952).

[5] J. R. Blake, J. Fluid Mech. 46, 199 (1971).

[6] M. Schmitt and H. Stark, Phys. Fluids 28, 012106 (2016).

[7] T. Ohmura, Y. Nishigami, A. Taniguchi, S. Nonaka, J. Manabe, T. Ishikawa, and M. Ichikawa, Proc. Natl. Acad. Sci. U.S.A. 115, 3231 (2018).

[8] Y. Nishigami, T. Ohmura, A. Taniguchi, S. Nonaka, J. Manabe, T. Ishikawa, and M. Ichikawa, Comm. Int. Biol. 11, e1506666 (2018).

[9] T. Vicsek and A. Zafeiris, Phys. Rep. 517, 71 (2012).

[10] C. C. Maass, C. Krüger, S. Herminghaus, and C. Bahr, Annu. Rev. Condens. Matter Phys. 7, 171 (2016).

[11] P.-G. de Gennes, F. Brochard-Wyart, and D. Quéré, Capillarity and Wetting Phenomena (Springer, New York, 2004).

[12] M. C. Marchetti, J. F. Joanny, S. Ramaswamy, T. B. Liverpool, J. Prost, M. Rao, and R. A. Simha, Rev. Mod. Phys. 85, 1143 (2013).

[13] S. Ramaswamy, Annu. Rev. Condens. Matter Phys. 1, 323 (2010).

[14] Y. Sumino, N. Magome, T. Hamada, and K. Yoshikawa, Phys. Rev. Lett. 94, 068301 (2005).

[15] R. Shimizu and H. Tanaka, Nat. Commun. 6, 7407 (2015).

[16] S. Katsura, A. Yamaguchi, N. Harada, K. Hirano, and A. Mizuno, in Conference Record of the 1999 IEEE Industry Applications Conference. Thirty-Forth IAS Annual Meeting (Cat. No.99CH36370) (IEEE, New York, 1999), Vol. 2, pp. 1124-1129, https://doi.org/10.1109/IAS.1999.801645. 
[17] E. Verneuil, M. L. Cordero, F. Gallaire, and C. N. Baroud, Langmuir 25, 5127 (2009).

[18] M. Ichikawa, F. Takabatake, K. Miura, T. Iwaki, N. Magome, and K. Yoshikawa, Phys. Rev. E 88, 012403 (2013).

[19] J. Čejková, M. Novák, F. Štěpánek, and M. M. Hanczyc, Langmuir 30, 11937 (2014).

[20] S. Thutupalli, R. Seemann, and S. Herminghaus, New J. Phys. 13, 073021 (2011).

[21] Z. Izri, M. N. van der Linden, S. Michelin, and O. Dauchot, Phys. Rev. Lett. 113, 248302 (2014).

[22] A. Izzet, P. G. Moerman, P. Gross, J. Groenewold, A. D. Hollingsworth, J. Bibette, and J. Brujic, Phys. Rev. X 10, 021035 (2020).

[23] B. V. Hokmabad, R. Dey, M. Jalaal, D. Mohanty, M. Almukambetova, K. A. Baldwin, D. Lohse, and C. C. Maass, Phys. Rev. X 11, 011043 (2021).

[24] C. de Blois, V. Bertin, S. Suda, M. Ichikawa, M. Reyssat, and O. Dauchot, Soft Matter 17, 6646 (2021).

[25] C. Krüger, G. Klös, C. Bahr, and C. C. Maass, Phys. Rev. Lett. 117, 048003 (2016).

[26] T. Yamamoto and M. Sano, Soft Matter 13, 3328 (2017).

[27] M. Suga, S. Suda, M. Ichikawa, and Y. Kimura, Phys. Rev. E 97, 062703 (2018).

[28] M. Morozov and S. Michelin, Soft Matter 15, 7814 (2019).

[29] F. Takabatake, N. Magome, M. Ichikawa, and K. Yoshikawa, J. Chem. Phys. 134, 114704 (2011).

[30] F. Takabatake, K. Yoshikawa, and M. Ichikawa, J. Chem. Phys. 141, 051103 (2014).

[31] K. H. Nagai, F. Takabatake, Y. Sumino, H. Kitahata, M. Ichikawa, and N. Yoshinaga, Phys. Rev. E 87, 013009 (2013).
[32] M. Morozov and S. Michelin, J. Chem. Phys. 150, 044110 (2019).

[33] S. Michelin, E. Lauga, and D. Bartolo, Phys. Fluids 25, 061701 (2013).

[34] See Supplemental Material at http://link.aps.org/ supplemental/10.1103/PhysRevLett.127.088005 for detailed description of the experimental setup, the results of supplementary experiments and the theoretical model.

[35] W. Thielicke and E. J. Stamhuis, J. Open Res. Software 2, e30 (2014).

[36] Y. P. Kalmykov and W. T. Coffey, Langevin Equation, The: With Applications To Stochastic Problems In Physics, Chemistry And Electrical Engineering (Fourth Edition), World Scientific Series In Contemporary Chemical Physics (World Scientific Publishing Company, Singapore, 2017).

[37] M. Morozov, Soft Matter 16, 5624 (2020).

[38] K. Holmberg, B. Jönsson, B. Kronberg, and B. Lindman, in Surfactants and Polymers in Aqueous Solution (John Wiley and Sons, Ltd, New York, 2002), Chap. 2, pp. 39-66, https://onlinelibrary.wiley.com/doi/book/10.1002/ 0470856424.

[39] Y.-C. Liao, O. A. Basaran, and E. I. Franses, AIChE J. 49, 3229 (2004).

[40] M. J. Comuñas, X. Paredes, F. M. Gaciño, J. Fernández, J. P. Bazile, C. Boned, J. L. Daridon, G. Galliero, J. Pauly, K. R. Harris, M. J. Assael, and S. K. Mylona, J. Phys. Chem. Ref. Data 42, 033101 (2013).

[41] A. Farutin, S. Rafaï, D. K. Dysthe, A. Duperray, P. Peyla, and C. Misbah, Phys. Rev. Lett. 111, 228102 (2013).

[42] N. P. Barry and M. S. Bretscher, Proc. Natl. Acad. Sci. U.S.A. 107, 11376 (2010).

[43] E. J. Campbell and P. Bagchi, Phys. Fluids 29, 101902 (2017). 